\title{
Blue Stragglers in Low-Luminosity Star Clusters ${ }^{1}$
}

\author{
Eric L. Sandquist \\ San Diego State University, Department of Astronomy, San Diego, CA 92182 \\ erics@mintaka.sdsu.edu
}

\begin{abstract}
We examine the blue straggler populations of 13 low-luminosity $\left(M_{V_{t}} \gtrsim-6\right)$ globular clusters and 2 old open clusters. These clusters test blue straggler formation in environments intermediate between higher luminosity (and usually higher density) clusters and the Galactic field. The anti-correlation between the relative frequency of blue stragglers $\left(F_{B S S}=N_{B S S} / N_{H B}\right)$ and cluster luminosity continues to the lowest luminosity clusters, which have frequencies meeting or exceeding that of field stars. In addition we find that the anti-correlation between straggler frequency and central density disappears for clusters with density less than about $300 L_{V, \odot} \mathrm{pc}^{-3}$, although this appears to be an artifact of the correlation between cluster luminosity and central density. We argue on observational (wide, eccentric binaries containing blue stragglers in M67, and the existence of very bright stragglers in most of the clusters in our sample) and theoretical grounds that stellar collisions still produce a significant fraction of the blue stragglers in low luminosity star clusters due to the long-term survival of wide binaries.
\end{abstract}

Subject headings: binaries: general — blue stragglers - globular clusters: general - open clusters and associations: individual (M67, NGC 188) - stars: luminosity function, mass function

Blue stragglers are one of several exotic stellar populations whose creation seems to be intimately tied to the dynamical environment that stars find themselves in. These stars are probably created when the mass of a normal main sequence star is increased by external means: the longest-surviving explanations involve either mass transfer within a binary system or the merger of stars in a binary or during a collision. The main debate is over

\footnotetext{
${ }^{1}$ Based on observations with the NASA/ESA Hubble Space Telescope, obtained at the Space Telescope Science Institute, which is operated by the Association of Universities for Research in Astronomy, Inc., under NASA Contract NAS 5-26555.
} 
which mechanisms play the largest roles in different environments. In the Galactic field at one extreme, the probability that two field stars (or binary systems) will strongly interact after they leave their birth environment is expected to be vanishingly small. Indeed, radial velocity surveys have identified a high fraction of single-line spectroscopic binaries with low eccentricity among field blue stragglers, which supports the idea that these stragglers were formed via binary mass transfer (Preston \& Sneden 2000; Carney et al. 2001, 2005).

At the other extreme are the most luminous $\left(M_{V_{t}}<-6\right)$ globular clusters. Piotto et al. (2004, hereafter P04) found a strong anti-correlation between blue straggler frequency [relative to horizontal branch (HB) stars] and integrated cluster magnitude, and a weaker anti-correlation with central density. (A similar relation can be seen in Ferraro et al. 1995.) Because expected collision rates correlate with cluster mass, Davies et al. (2004) proposed that primordial binaries that would form stragglers were being depleted earlier in the history of a massive cluster, thanks to exchanges of massive stars into the binaries and subsequent mass transfer. For the most massive clusters $\left(M_{V_{t}} \lesssim-8.8\right)$, they predicted that star collisions would take over as the dominant mechanism for forming stragglers.

Regardless of the interpretation of the P04 results, the connection between the field and cluster environments has not been made. To this end, we have examined low-mass globular clusters and old open clusters to see whether the straggler populations still show the influences of the cluster environment, or whether that influence has begun to disappear.

\section{Observational Material}

To collect the largest possible samples of blue stragglers and comparison stars for lowluminosity globular clusters, we used high quality ground-based photometry from the literature and photometry from the Hubble Space Telescope (HST) archives (all using the WFPC2 camera). In all cases, we chose datasets with little photometric scatter at the turnoff, so that contamination of the blue straggler sample by normal main sequence stars was minimal. All HST data was reduced in a uniform manner using the HSTPHOT package (Dolphin 2000a).

Our final sample consists of 13 globular clusters with $M_{V_{t}}>-6$ (9 with HST data). Color-magnitude diagrams (CMDs) for the primary HST fields are shown in Fig. 1. The blue stragglers discussed here are all brighter than the cluster turnoff and bluer (by 0.05 in $(B-V))$, and there was typically no difficulty with field star contamination of the portion of the CMD populated by stragglers. Although most of our straggler identifications are based on $V-I$ colors (as opposed to $B-V$ for P04), there were few stars for which the choice of color could affect the selection. In some clusters, a significant field star population 
redder than the cluster turnoff and brighter than the subgiant branch made it impractical to use cluster giants as a comparison population, so we used HB stars instead. Clusters with large field contamination could sometimes be used if they had blue HB morphologies. The complete sample of blue stragglers and HB stars will be presented in a companion paper.

We also examined the blue straggler populations of two well-studied old open clusters: M67 and NGC 188. Both of these clusters have extensive proper motion membership information available that allows us to identify stragglers, and to estimate the absolute magnitude of the cluster by summing the fluxes of member stars $\left(M_{V_{t}} \approx-3.9\right.$ and -3.8 , respectively). We have also estimated the central luminosity densities ( $\log \rho_{0} \sim 2.3$ and 2.2 , respectively) using structural information from Bonatto \& Bica (2003) and Bonatto et al. (2005). Although these clusters have lower ages (and more luminous stars on average $^{2}$ ), these two clusters have characteristics comparable to the faintest globular clusters.

P04 used two measures to judge the relative frequency of blue stragglers in a cluster: the population ratio $F_{B S S}^{H B}=N_{B S S} / N_{H B}$, and the number of stragglers per absolute visual flux unit $N_{S}^{B S S}$. In Fig. 2, we plot $F_{B S S}^{H B}$ versus absolute cluster magnitude and central density. Values for these cluster properties were taken from the compilation of Harris (1996). With the inclusion of data for these 13 clusters, we extend the cluster sample fainter about 3 magnitudes in $M_{V_{t}}$ and more than 3 orders of magnitude in central density. The significant anti-correlation between $F_{B S S}^{H B}$ and $M_{V_{t}}$ seen by P04 continues to the low luminosity end of the sample. That end is anchored by the clusters E $3\left(M_{V_{t}}=-2.77\right)$ and Palomar 13 $\left(M_{V_{t}}=-3.74\right)$. E 3 has no known HB stars (one unconfirmed candidate), and so produces a lower limit. The majority of the clusters have higher relative frequencies than all but a handful of the clusters in the P04 sample. (We also examined the cluster M71, which was the faintest cluster in the P04 data, to verify that our straggler selection produced a straggler frequency equal to theirs to within the errors.) None of the clusters examined here has $F_{B S S}^{H B}<0.72$, which is a noticeable departure from the behavior of more luminous clusters in spite of the significant variations from cluster to cluster.

Although the open clusters that we have been able to include are significantly younger than the globular clusters in the sample (4 Gyr for M67, 6 Gyr for NGC 188), they appear to follow the relation defined by the globular clusters, and agree with the high value found for Pal 13. We have used red clump stars (in NGC 188, including two blue subdwarfs) as the comparison populations for the open clusters, but this should not be an issue since the lifetimes of stars in this phase is within about $10 \%$ of the lifetimes of red HB stars in

\footnotetext{
${ }^{2}$ Since cluster luminosity and luminosity density are being used as stand-ins for cluster mass or central mass density, the open cluster points should be offset toward lower luminosity and density.
} 
globular clusters (Girardi et al. 2000). This puts the open cluster straggler populations in a new context - normal for their total masses, not abnormally large.

\section{Relation to Field Blue Stragglers and Open Clusters}

Because our sample of low-luminosity clusters also contains some of the lowest density clusters, we compare to the population of field blue stragglers (Carney et al. 2005, 2001; Preston \& Sneden 2000). The prevalence of single-lined low-eccentricity spectroscopic binaries in the field sample implies that stable binary mass transfer is the main production mechanism among field stars. (Coalescence of close binaries like W UMa systems would produce the rest.) The 4 lowest-luminosity clusters in our sample are known to have binary fractions comparable to that of field stars, based on precise photometry of the main sequence $\left[f_{b}>0.30 \pm 0.04\right.$ for Pal 13 (Clark et al. 2004); $f_{b}>0.29 \pm 0.09$ for E3 (Veronesi et al. 1996); $f_{b}>0.38$ for M67 (Montgomery et al. 1993); $f_{b} \gtrsim 0.5$ for NGC 188 (von Hippel \& Sarajedini 1998)]. Preston \& Sneden (2000) found that low-luminosity $\left(M_{V_{t}}>-6\right)$ globular clusters have larger $F_{B S S}^{H B}$ values than higher luminosity clusters and estimated that $F_{B S S}^{H B} \approx 4$ in the field. We find that only the four least luminous clusters have BSS frequencies that are consistent with (Palomar 13, M67, NGC 188) or exceed (E3) the Preston \& Sneden estimate for the field. Thus it seems that all but the least luminous globular clusters are less efficient at producing stragglers than the field.

Binary systems that transfer mass are quite "hard" systems with binding energies that are considerably larger than kinetic energies of the stars they encounter in most clusters (Davies et al. 2004). A figure of merit is $\eta=G m_{1} m_{2} / a \sigma^{2}\langle m\rangle$, where $m_{1}, m_{2}$, and $\langle m\rangle$ are masses of the binary components and the average single star, $a$ is the binary separation, and $\sigma$ is the cluster velocity dispersion (Ivanova et al. 2005). The boundary between "hard" and "soft" binaries is $\eta \approx 1$, while mass transfer binaries in the clusters described here have $\eta \gtrsim 100$. In general, such hard binaries should survive in a cluster and produce stragglers via mass transfer. Do stellar collisions still produce stragglers though?

For the nearby open clusters, studies of individual blue stragglers provide information on the formation mechanisms. In NGC 188, the straggler PKM 4330 has a high membership probability $\left(P_{\mu}=82 \%\right.$; Platais et al. 2003), but stands more than 3 magnitudes above the turnoff magnitude, which probably means it has more than twice the turnoff mass and results from a multi-star collision. There is a much larger body of information on M67 stars. Like PKM 4330, the brightest straggler (S977) appears to have a mass more than twice the cluster turnoff mass. There are many variable blue stragglers, including S1036 (W UMa variable), S1082 (RS CVn variable, X-ray source, possible triple system containing two 
blue stragglers), and S1280 and S1284 ( $\delta$ Scuti pulsators, S1284 in a short-period eccentric binary). Several more blue stragglers are in long-period spectroscopic binaries: S752, S975, and S1195 (low-eccentricity orbits, S975 with hot companion), and S1267 and S997 (eccentric orbits). Roughly half of these systems probably formed via normal binary mass transfer (S752, S975, S1036, and S1195), but an equally large number of systems probably require a collisional mechanism involving more than 2 stars (most notably, S977, S997, S1082, S1267, and $\mathrm{S} 1284)^{3}$.

\section{Relation to Higher Luminosity Clusters}

The clusters in this study reveal a continuation of the strong anti-correlation between straggler frequency and cluster luminosity seen by P04, and a break in the weaker anticorrelation with cluster density (lower panel of Fig. 2. An important question is whether the dependence on cluster density has physical meaning or whether it is an artifact of a correlation between cluster properties. Fig. 3 shows the relation between integrated magnitude and central density (Harris 1996). The similarity of this plot and the bottom panel of Fig. 2 leads us to believe that the density relationship is an artifact - the clusters E3 and Pal 13 most clearly stand out as clusters with high straggler frequencies, low luminosity, and relatively high central densities. Low-luminosity clusters $\left(-6<M_{V_{t}}<-4\right)$ cover almost the entire range of central densities, while straggler frequencies remain uniformly high. We must keep in mind that for most of the clusters surveyed by P04 the blue straggler samples are mostly confined to the core, and selection effects in the P04 cluster sample (aside from $\left.M_{V_{t}}>-6\right)$ may influence the interpretation of these figures. However, central density is not as good a predictor of the size of the straggler population as integrated cluster luminosity.

Does this mean that strong gravitational interactions between stars are unimportant in the production of stragglers in low-luminosity clusters? As mentioned in $§ 2$, a significant fraction of stragglers in M67 are in binaries showing evidence of strong multiple-star interactions. Stars at the bright end of the straggler luminosity function should also reflect the relative importance of stellar collisions and binary coalescences since mass transfer is very unlikely to be $100 \%$ efficient during the formation of a straggler in a wide binary. As shown in Fig. 1, the clusters in our low-luminosity sample still produced a substantial fraction of stragglers at the high luminosity end (more than 10\% have $V-V_{T O}<-1.75$ ). 9 of the 13

\footnotetext{
${ }^{3}$ Because M67 is the youngest cluster discussed here ( 4.0 Gyr; VandenBerg \& Stetson 2004), we must keep in mind that these stragglers would have evolved and died by the time the cluster's age reached that of a globular cluster $(\sim 12 \mathrm{Gyr})$.
} 
globular clusters (as well as M71) and both open clusters have at least one straggler more than 2 magnitudes brighter than the turnoff, implying a collision of at least three stars.

We can attempt to qualitatively understand the problem by examining the timescale for collisions $t_{\text {coll }}$ (Ivanova et al. 2005; Davies et al. 2004). The dominant factor in $t_{\text {coll }}$ is number density $\left(t_{\text {coll }} \propto n^{-1}\right)$. Our cluster sample covers more than three orders of magnitude in central density, and when combined with the sample of P04, the total range is more than six orders of magnitude. So, it might be tempting to believe that the near constancy of $F_{B S S}^{H B}$ for many clusters with $\log \rho_{0} \lesssim 2.5$ is an indication that primordial binary star mechanisms dominate and strong interactions have become unimportant.

The velocity dispersion $\sigma$ is also a few times lower for low-luminosity clusters compared to most clusters in the P04 sample: from a maximum of $2.4 \mathrm{~km} \mathrm{~s}^{-1}$ for NGC 6535 (Pryor \& Meylan 1993) to $0.6-0.9 \mathrm{~km} \mathrm{~s}^{-1}$ for Palomar 13 (Blecha et al. 2004) and $0.8 \mathrm{~km} \mathrm{~s}^{-1}$ for M67 (Girard et al. 1989), to $0.1-0.4 \mathrm{~km} \mathrm{~s}^{-1}$ for Palomar 5 (Odenkirchen et al. 2002). Low encounter velocities increase the time between interactions but more strongly increase the probability of collision via gravitational focusing, so that $t_{\text {coll }} \propto \sigma$ (Binney \& Tremaine 1987). Velocity dispersion only varies by a factor of about 100 among all Milky Way globular clusters, so its effects on the timescale are usually overwhelmed by those of cluster density.

The velocity dispersion has a larger effect on the statistical properties of the binary star population though. As velocity dispersion decreases, wider binaries qualify as "hard" $\left(\eta \propto \sigma^{-2}\right)$. For $\sigma=10 \mathrm{~km} \mathrm{~s}^{-1}$, the hard-soft boundary is already at several AU. For most globular clusters (and all of the ones in the present sample), binary systems that would ultimately produce blue stragglers via mass transfer in a giant evolutionary phase are likely to survive due to their hardness, even though collisions are fairly frequent. For the moment, we only consider collisions (i.e. strong gravitational interactions) involving binaries, and not physical collisions of stars. In a cluster like NGC 6535 at the bright end of our sample, the collision timescale for the largest binaries that transfer mass in the giant phase implies that they have a few collisions in the age of the cluster. (Keep in mind that these collision timescales use central densities, and so are overestimates for the cluster as a whole.)

For lower luminosity clusters, there are competing effects. Lower velocity dispersion allows wider binaries to survive to the present day. Photometric measurements of large binary fractions among some of the low-luminosity clusters in our sample (Clark et al. 2004; Veronesi et al. 1996; Montgomery et al. 1993; von Hippel \& Sarajedini 1998) support this. Lower cluster density increases the collision timescale for binaries of a given size, although this is partly offset by lower $\sigma$ and increased gravitational focussing. However, the changes in the nature of the binary population (increased binary fraction and increased average orbital separation) help enhance the overall binary collision rate. 
The collision timescale for binaries at the hard-soft boundary has $t_{\text {coll }} \propto n^{-1} \sigma^{3}$, which allows lower velocity dispersion to partially cancel the effects of lower number density. If we assume that the present-day binary period distribution is uniform in $\log _{10} P$ between a minimum period (set by star sizes) and the hard-soft boundary, then the typical binary involved in a strong interaction will have $a \approx a_{h s} / \ln \left(a_{h s} / a_{\min }\right)$, where $a_{\min }$ and $a_{h s}$ are orbital separations of the hardest binaries and the hard-soft boundary, respectively. Thus, the collision timescale for these systems should only be a factor of $5-15$ more than the collision timescale for binary systems at the hard-soft boundary.

A strong interaction involving a close binary is very likely to produce at least one physical collision of stars $\left(\sigma_{\text {coll }} \sim \pi\left(a_{1}+a_{2}\right)^{2}\left(v_{c} / v_{\infty}\right)^{2}\right.$, where $v_{c}$ is the critical velocity needed to unbind the binary; Fregeau et al. 2004). However, for wide but hard binaries in low-mass clusters, the main sequence star components present small targets compared to the binary. For a ratio of stellar radius to orbital separation $R / a \lesssim 0.001$, the cross section for collisions decreases approximately as $(R / a)^{0.75}$, according to Figure 9 of Fregeau et al. (2004). Because the binary collision timescale $t_{\text {coll }} \propto a^{-1}$, the timescale for stellar collisions involving binaries then decreases slowly with increasing $a$.

\section{Conclusions}

A combination of effects may therefore conspire to produce a significant number of stellar collisions involving binaries even within low luminosity clusters. The lack of a stronger correlation between $F_{B S S}^{H B}$ and central density may result from the effects of changing velocity dispersion and binary population. This might also partially explain the apparent lack of correlation between $F_{B S S}^{H B}$ and the probability $\Gamma_{*}$ that a given star will have a collision in one year (P04) since the calculation of $\Gamma_{*}$ from King models does not account for changes in the binary star distributions between clusters.

Populations of low-luminosity X-ray sources in globular clusters [such as quiescent lowmass X-ray binaries (qLMXBs), cataclysmic variables, and millisecond pulsars] appear to behave differently. Pooley et al. (2003) found strong correlations between number of sources and normalized encounter rate $\Gamma$, and Heinke et al. (2003) identified probable qLMXBs that are consistent with predictions based on tidal capture or exchanges during multiple star interactions. It is not clear that this is consistent with the scenario of Davies et al. (2004) in which massive stars exchange into previously-existing binaries in the most massive clusters, forming stragglers that die off before the present day. The blue straggler and low-luminosity X-ray populations may be giving us complimentary information about the importance of collisions and the prevalence of binaries in star clusters. 
We suggest two directions to disentangle the important factors driving the dynamical evolution of clusters. First, the binary star populations of more clusters need to be observationally characterized - particularly the binary fraction and the period distribution. Second, models of cluster evolution that examine the production of stragglers and X-ray sources are needed. The recent hybrid models (binary population synthesis with dynamics) of Ivanova et al. (2005) provide some guidance, although they modelled clusters that are denser and more massive than the ones in our sample. They find that the present-day core binary fraction is inversely related to cluster density, and that the binary period distribution remains nearly flat in $\log P$ up to periods of $10^{4} \mathrm{~d}$ and more for their lowest-density clusters. The low-luminosity clusters examined in this study make perfect subjects for numerical studies of cluster dynamics with current generations of computer codes.

We would like to thank the anonymous referee for helpful suggestions, K. Cudworth, D. Dinescu, B. Mochejska, A. Rosenberg, and A. Sarajedini for copies of their datasets, and useful conversations with O. De Marco and J. Fregeau. This work has been funded through grant AST 00-98696 from the National Science Foundation to E.L.S. and M. Bolte. 


\section{REFERENCES}

Binney, J. \& Tremaine, S. 1987, Galactic Dynamics (Princeton University Press, Princeton)

Blecha, A., Meylan, G., North, P., \& Royer, F. 2004, A\&A, 419, 533

Bonatto, C., \& Bica, E. 2003, A\&A, 405, 525

Bonatto, C., Bica, E., \& Santos, J. F. C. 2005, A\&A, 433, 917

Carney, B. W., Latham, D. W., Laird, J. B., Grant, C. E., \& Morse, J. A. 2001, AJ, 122, 3419

Carney, B. W., Latham, D. W., \& Laird, J. B. 2005, AJ, 129, 466

Clark, L. L., Sandquist, E. L., \& Bolte, M. 2004, AJ, 128, 3019

Davies, M. B., Piotto, G., \& de Angeli, F. 2004, MNRAS, 349, 129

Dolphin, A. E. 2000a, PASP, 112, 1383

Ferraro, F. R., Fusi Pecci, F., \& Bellazzini, M. 1995, A\&A, 294, 80

Fregeau, J. M., Cheung, P., Portegies Zwart, S. F., \& Rasio, F. A. 2004, MNRAS, 352, 1

Girard, T. M., Grundy, W. M., Lopez, C. E., \& van Altena, W. F. 1989, AJ, 98, 227

Girardi, L., Bressan, A., Bertelli, G., \& Chiosi, C. 2000, A\&AS, 141, 371

Harris, W. E. 1996, AJ, 112, 1487

Heinke, C. O., Grindlay, J. E., Lugger, P. M., Cohn, H. N., Edmonds, P. D., Lloyd, D. A., \& Cool, A. M. 2003, ApJ, 598, 501

Ivanova, N., Belczynski, K., Fregeau, J. M., \& Rasio, F. A. 2005, MNRAS, 358, 572

Montgomery, K. A., Marschall, L. A., \& Janes, K. A. 1993, AJ, 106, 181

Odenkirchen, M., Grebel, E. K., Dehnen, W., Rix, H.-W., \& Cudworth, K. M. 2002, AJ, 124,1497

Piotto, G., et al. 2004, ApJ, 604, L109 (P04)

Platais, I., Kozhurina-Platais, V., Mathieu, R. D., Girard, T. M., \& van Altena, W. F. 2003, AJ, 126,2922 
Pooley, D., et al. 2003, ApJ, 591, L131

Preston, G. W., \& Sneden, C. 2000, AJ, 120, 1014

Pryor, C., \& Meylan, G. 1993, in ASP Conf. Ser. 50, Structure and Dynamics of Globular Clusters, eds. S. G. Djorgovski and G. Meylan, (ASP: San Francisco), 357

VandenBerg, D. A., \& Stetson, P. B. 2004, PASP, 116, 997

Veronesi, C., Zaggia, S., Piotto, G., Ferraro, F. R., \& Bellazzini, M. 1996, in ASP Conf. Ser. 92, Formation of the Galactic Halo...Inside and Out, ed. H. Morrison \& A. Sarajedini (San Francisco: ASP), 301

von Hippel, T., \& Sarajedini, A. 1998, AJ, 116, 1789 


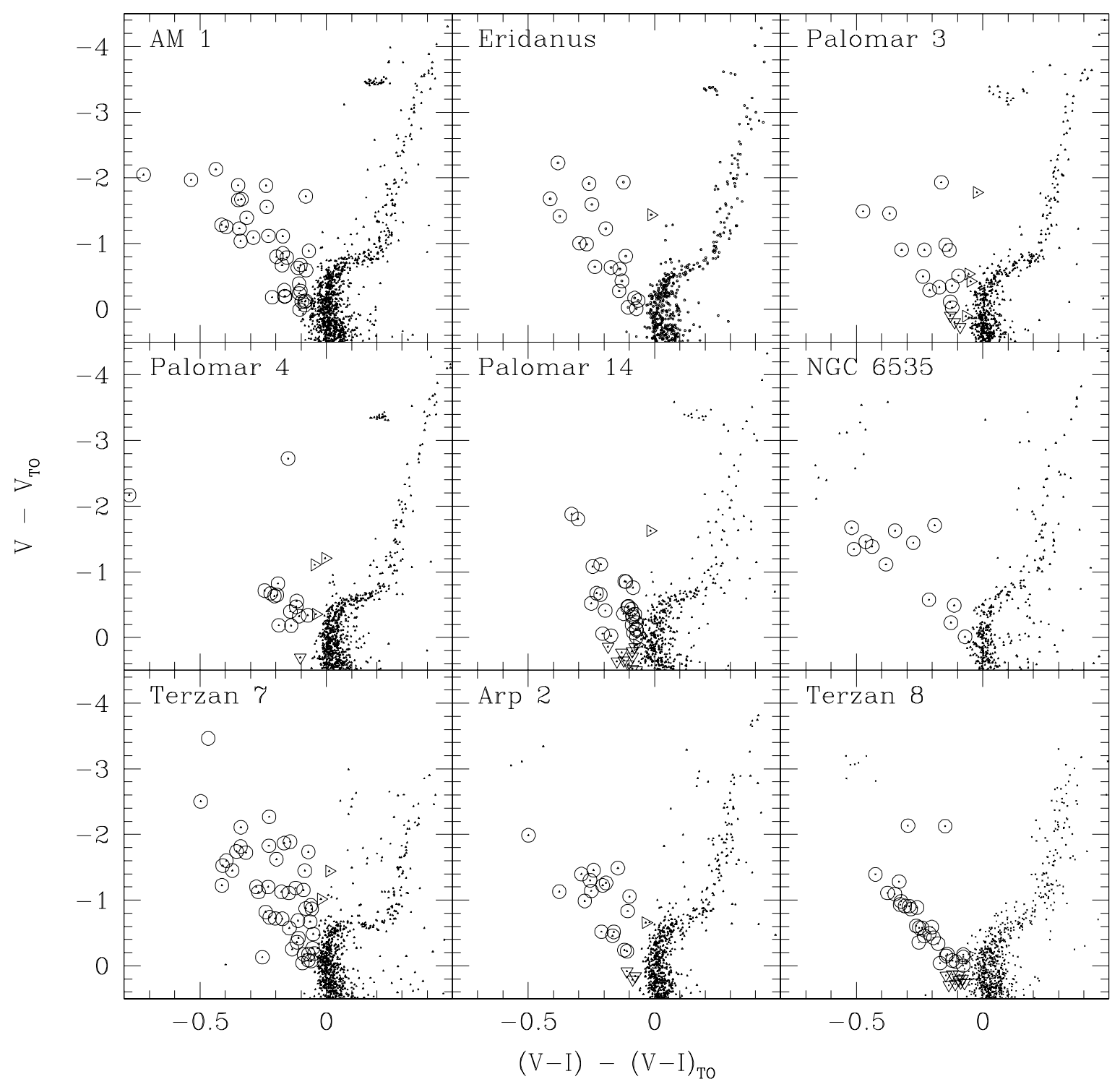

Fig. 1.- CMDs for 9 globular clusters with HST photometry. Open circles are stragglers used in this study, while open triangles show candidates too red or too faint for selection. For several of these clusters, additional photometry was used to identify other stragglers outside the primary field. 

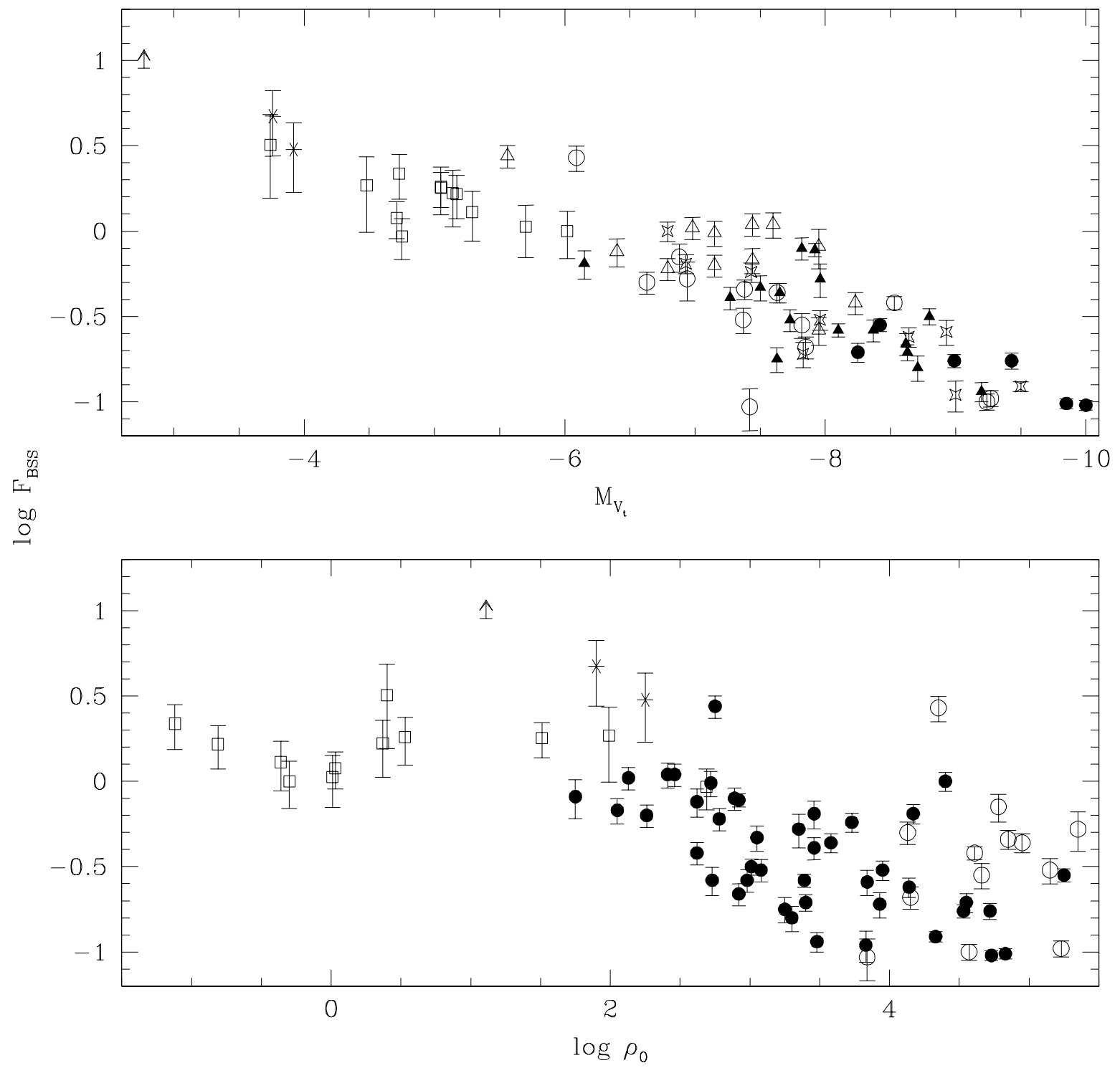

Fig. 2.- Relative frequencies of blue stragglers as a function of cluster absolute magnitude and central density. Open squares are globular clusters and asterisks are open clusters from this study, while all other points are from the HST Snapshot survey of Piotto et al. (2004). Open circles are post-core-collapse clusters. In the left panel, other symbols represent clusters in different ranges of central density: $\log \rho_{0}<2.8$ : open triangles; $2.8<\log \rho_{0}<3.6$ : filled triangles; $3.6<\log \rho_{0}<4$.4: stars; $\log \rho_{0}>4$.4: filled circles. 


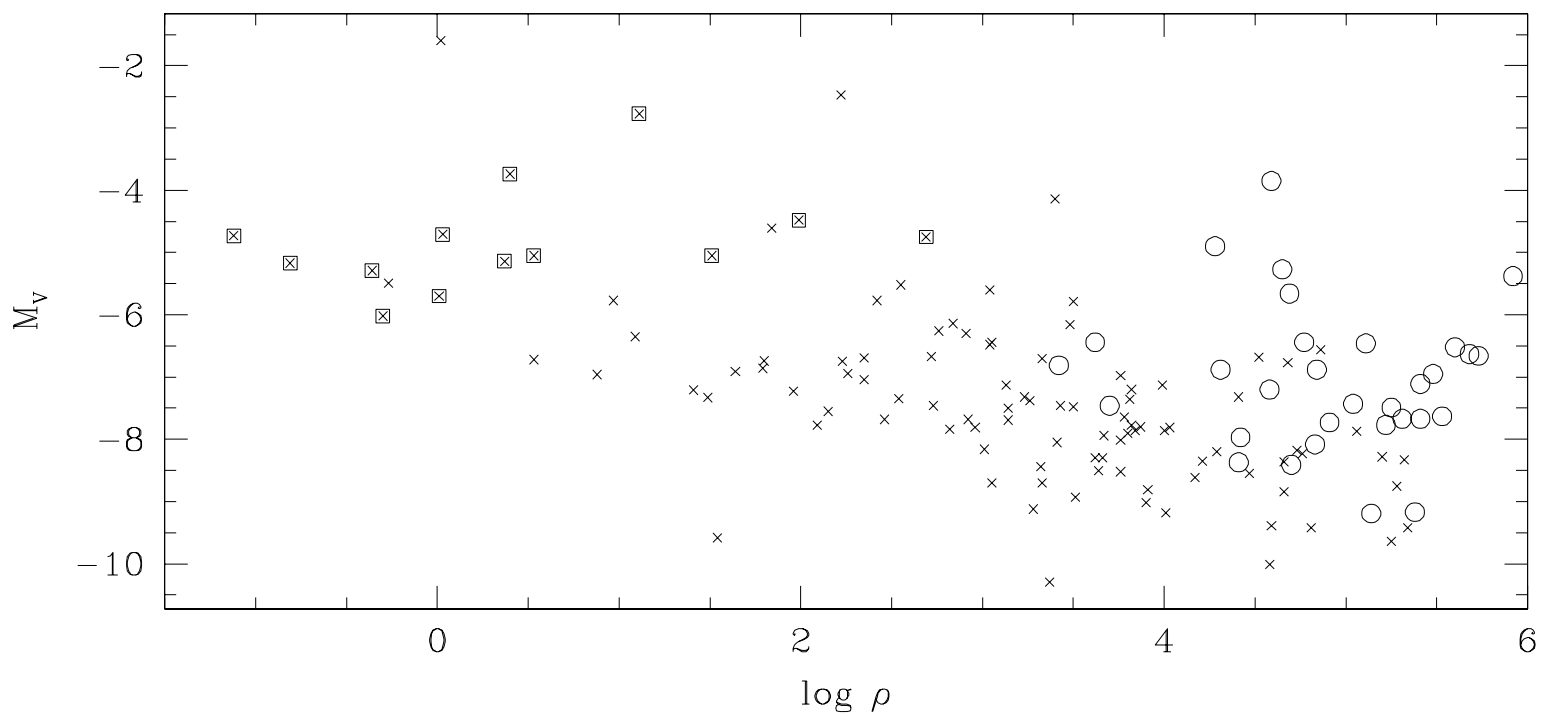

Fig. 3.- Cluster absolute magnitude versus central density for Galactic globular clusters (Harris 1996). Open circles are post-core-collapse clusters, and open squares are globular clusters in this study. Central density is in $L_{V, \odot} \mathrm{pc}^{-3}$. 